\title{
OPTIMAL DECODING ORDER UNDER TARGET RATE CONSTRAINTS
}

\author{
Alberto Suárez ${ }^{1}$,Mérouane Debbah ${ }^{2}$,Laura Cottatellucci ${ }^{1}$ and Eitan Altman ${ }^{3}$ \\ ${ }^{1}$ Institut Eurecom, Sophia Antipolis, France suarezr,cottatellucci@eurecom.fr \\ ${ }^{2}$ Supelec, Gif sur Yvette, France merouane.debbah@supelec.fr \\ ${ }^{3}$ INRIA, Sophia Antipolis, France eitan.altman@sophia.inria.fr
}

\begin{abstract}
In this contribution, we derive the optimal power allocation under target rate constraints in the case of an uplink multiuser system. Using asymptotic results based on random matrix theory, we provide a unified framework for determining the optimal decoding order when using Successive Interference Cancellation (SIC) receivers, namely the MMSE (Minimum Mean Square Error) and Matched filter SIC. Moreover, for decentralized systems, we show that each user can, under certain conditions, solely determine the power allocation based only on his channel energy and the statistics of the other users. Simulations in the case of finite systems validate the asymptotic claims.
\end{abstract}

\section{INTRODUCTION}

With the increasing importance of multi-user communication systems, one of the main issues is the satisfaction of the different users' rates under multi-user interference. In its full generality, this problem can be solved through proper power allocation (when the rate regions are achievable) [1, 2, 3]. However, the power allocation scheme depends on the channel realizations of all users, the type of receiver structure as well as the requested rates, as devised in recent contributions $[4,5,6]$. Moreover, the power allocation algorithm's complexity increases with the number of users as one has to solve a set of non-linear equations.

In the case of i.i.d signatures, [7] [8] derived, using results from asymptotic matrix theory, the optimal (which minimizes the total power) decoding order for MMSE-SIC receivers for a given set of requested rates. The power allocation was obtained explicitly and was shown to be independent of the channel realizations.

In this contribution, more general type of signatures are considered (Kronecker model for MIMO systems, CDMA in the case of frequency selective channels) and the decoding order and power allocation in the case of the MMSE-SIC is derived. For comparison purposes, similar results are derived for the matched filter SIC.

This work is supported in part by the FP6 project Cooperative and Opportunistic Communications in Wireless Networks (COOPCOM), Project Number: FP6-033533
Interestingly, it is shown that the power allocation (in the case of i.i.d signatures) can be determined in a decentralized manner (each user can determine his decoding order and power allocation based only on the knowledge of the discrete set of possible rates, whereas in general, the base station computes the algorithm and allocates the powers) for a high number of users in the network. This interesting result can be applied to reduce the downlink signalling of multi-user systems.

In section 2, the system model is presented. Section 3 introduces the receivers of interest and their respective SINR expressions. In section 4, the optimal decoding orders for matched filter SIC and MMSE-SIC are derived, while in section 5 the decentralized allocation scheme is introduced. Finally, in section 6 simulation results are shown to validate the asymptotic claims for finite systems.

\section{SYSTEM MODEL}

A system composed of a base station, with $N$ dimensions and $K$ users, is considered. We are interested in the uplink scenario. Each user $k$ is supposed to send a signal at a requested rate $R_{k}$. The input output relationship of the system is then given by:

$$
\mathbf{y}=\mathbf{H} \mathbf{P}^{\frac{1}{2}} \mathbf{S}+\mathbf{n}
$$

where $\mathbf{y}, \mathbf{s}, \mathbf{n}, \mathbf{H}$ and $\mathbf{P}^{\frac{1}{2}}$ are respectively the received signal, transmitted signal, additive white Gaussian noise (AWGN) of variance $\sigma^{2}$, the mixing matrix, and diagonal matrix of transmitted powers. In the following, these terms are written out as: $\mathbf{y}=\left[y_{1}, y_{2}, \ldots, y_{N}\right]^{T}, \mathbf{s}=\left[s_{1}, s_{2}, \ldots, s_{K}\right]^{T}$, $\mathbf{n}=\left[n_{1}, n_{2}, \ldots, n_{N}\right]^{T}$,

$$
\mathbf{H}=\left[\begin{array}{cccc}
h_{11} & h_{12} & \ldots & h_{1 K} \\
h_{21} & h_{22} & \ldots & h_{2 K} \\
\vdots & \vdots & \ddots & \vdots \\
h_{N 1} & \ldots & \ldots & h_{N K}
\end{array}\right]
$$

and

$$
\mathbf{P}^{\frac{1}{2}}=\left[\begin{array}{ccccc}
p_{1}^{\frac{1}{2}} & 0 & 0 & \ldots & 0 \\
0 & p_{2}^{\frac{1}{2}} & 0 & \ldots & 0 \\
\vdots & \vdots & \vdots & \ddots & 0 \\
0 & 0 & 0 & \cdots & p_{K}^{\frac{1}{2}}
\end{array}\right] .
$$


The $h_{i k}$ are independent zero mean gaussian variables with variances $\frac{\left|g_{i k}\right|^{2}}{N}$. In particular, the mixing matrix can be written as

$$
\mathbf{H}=\mathbf{G} \odot \mathbf{W}
$$

where $\mathbf{W}$ and $\mathbf{G}$ are respectively an $N \times K$ i.i.d. zero mean Gaussian matrix and the pattern mask specific to a given technology $\mathbf{G}=\left[g_{i k}\right]_{i=1 \ldots N, k=1 \ldots K} . \odot$ is the Hadamard product. The model is broad enough to incorporate several technologies: for example,

- OFDM with $g_{i k}=0$ if $k \neq i$.

- MIMO with Kronecker model [9], which can be shown to be equivalent, via unitary transforms to $g_{i k}=a_{i} b_{k}$.

- (MC)-CDMA systems with frequency selective channels: $g_{i k}$ represents the frequency response of user $k$ on carrier $i$.

In the following, column $\mathbf{h}_{k}$ corresponding to user $k$ will be called a signature irrespective of the technology.

\section{ASYMPTOTIC SINR FOR MULTI-USER RECEIVERS}

In this section, results for asymptotic random matrix theory are used to derive the SINR of two multi-user receivers, namely the matched filter SIC and the MMSE SIC. The successive interference cancellation receiver is based on a sequential estimation and substraction of the symbols. At each step, all the users being decoded are retrieved from the received signal, which reduces the interference for the next estimation step. In order to derive the expressions, let us consider user $k$, for which previous users $1, \ldots, k-1$ have already been decoded, and their interference cancelled. Denote by $\mathbf{h}_{\mathbf{k}}, \mathbf{g}_{\mathbf{k}}$ and $\mathbf{w}_{\mathbf{k}}$ the k-th column of the matrices $\mathbf{H}, \mathbf{G}$ and $\mathbf{W}$.

\subsection{Matched filter SIC receiver}

The matched filter for user $k$ is given by $\mathbf{u}_{k}^{H}=\mathbf{h}_{k}^{H}=\left(\mathbf{g}_{k} \odot\right.$ $\left.\mathbf{w}_{k}\right)^{H}$. The signal at the output of the matched filter is given by

$$
\mathbf{u}_{k}^{H} \mathbf{y}=\mathbf{p}_{\mathbf{k}}^{\frac{1}{2}}\left|\mathbf{g}_{\mathbf{k}} \odot \mathbf{w}_{\mathbf{k}}\right|^{\mathbf{2}} \mathbf{s}_{\mathbf{k}}+\sum_{\mathbf{i} \neq \mathbf{k}} \mathbf{u}^{\mathbf{H}} \mathbf{p}_{\mathbf{i}}^{\frac{1}{2}}\left(\mathbf{g}_{\mathbf{i}} \odot \mathbf{w}_{\mathbf{i}}\right) \mathbf{s}_{\mathbf{i}}+\mathbf{u}^{\mathbf{H}} \mathbf{n}
$$

and the SINR can be expressed as

$$
\frac{p_{k}\left(\sum_{i=1}^{N}\left|w_{i k}\right|^{2} g_{i k}^{2}\right)^{2}}{\sigma^{2}\left(\sum_{i=1}^{N}\left|w_{i k}\right|^{2} g_{i k}^{2}\right)+\sum_{l=k+1}^{K} p_{l}\left|\sum_{i=1}^{N} w_{i k}^{*} w_{i l} g_{i k} g_{i l}\right|^{2}} .
$$

In the case of a large number of users and dimensions increasing at the same rate (i.e $N, K \rightarrow \infty$ but the ratio $\frac{K}{N}=\alpha$, also known as the load of the system), and taking into account that the $w_{i j}$ are independent and $E\left\{\left|w_{i j}\right|^{2}\right\}=1 / N$ and $E\left\{\left|w_{i j}\right|^{4}\right\}=\frac{1}{N^{\alpha}} \quad$ with $\quad \alpha>1$, the SINR $\gamma_{k}$ can be shown to be equal to

$$
\gamma_{k}=\frac{p_{k}\left(\sum_{i=1}^{N} g_{i k}^{2}\right)^{2}}{N \sigma^{2} \sum_{i=1}^{N} g_{i k}^{2}+\sum_{l=k+1}^{K}\left(p_{l} \sum_{i=1}^{N} g_{i k}^{2} g_{i l}^{2}\right)}
$$

\subsection{MMSE-SIC receiver}

The MMSE-SIC is based on the linear MMSE receiver, known for being the linear multi-user detection filter which maximized the output SINR and given by:

$$
\mathbf{u}_{k}^{H}=\mathbf{h}_{k}^{H}\left(\sum_{l=k}^{K} p_{l} \mathbf{h}_{l} \mathbf{h}_{l}^{H}+\sigma^{2} \mathbf{I}_{N}\right)^{-1}
$$

After some manipulations, the SINR can be shown to be equal to:

$$
\gamma_{k}=p_{k} \mathbf{h}_{k}^{H}\left(\sum_{l=k+1}^{K} p_{l} \mathbf{h}_{l} \mathbf{h}_{l}^{H}+\sigma^{2} \mathbf{I}_{\mathbf{N}}\right)^{-\mathbf{1}} \mathbf{h}_{\mathbf{k}}
$$

The output SINR depends in an intricated manner on the different signature realizations. Interestingly, when the dimensions of the system increase at the same rate (i.e $N, K \rightarrow$ $\infty, \frac{K}{N}=\alpha$ ), it can be shown [10] and [11] that the SINR, $\gamma_{k}$, at the output of the MMSE-SIC receiver is given by:

$$
\gamma_{k}=\frac{p_{k}}{N} \sum_{i=1}^{N} \frac{\left|g_{i k}\right|^{2}}{\sigma^{2}+\frac{1}{N} \sum_{l=k+1}^{K} \frac{p_{l}\left|g_{i l}\right|^{2}}{1+\gamma_{l}}}
$$

Hence, the SINR does not depend on the channel realization due to the averaging effects.

\section{DECODING ORDER ANALYSIS}

A major problem in successive interference cancellation is to determine the decoding order and the power of the users for a given target rate which minimizes the total power $\sum_{i=1}^{K} p_{i}$. Hence, if we assume that the users have requested target rates $R_{k}$ for which the target SINR $\gamma_{k}$ is given by (assuming Gaussian symbols) $\gamma_{k}=2^{R_{k}}-1$, the central entity has to solve the set of non-linear equations 2 and 3 , with the power constraint. This is not a trivial problem and moreover, only iterative techniques with no proof of convergence are available.

\subsection{Matched filter receiver}

In this section a separable model will be considered for the channel energy profiles, i.e. $g_{i k}=a_{i} b_{k}$, which encompasses 
MIMO and frequency selective CDMA systems, among others. Hence equation (2) can be rewritten as

$$
\gamma_{k}=\frac{p_{k} b_{k}^{2}\left(\sum_{i=1}^{N} a_{i}^{2}\right)^{2}}{N \sigma^{2} \sum_{i=1}^{N} a_{i}^{2}+\sum_{l=k+1}^{K}\left(p_{l} b_{l}^{2} \sum_{i=1}^{N} a_{i}^{4}\right)}
$$

In the following, define $E_{k}=\frac{1}{N} \sum_{i=1}^{N}\left|g_{i k}\right|^{2}$ as the average energy of user $k$, then the following result holds:

Result: For the matched filter SIC receiver, the optimal decoding order is given in order of decreasing channel energies, i.e. $E_{1}>E_{2}>\cdots>E_{K}$ (where the index denotes the decoding order of the user), and the power allocation to satisfy the requested rates is given by

$$
p_{k}=\frac{\gamma_{k}}{E_{k}} \prod_{l=k+1}^{K}\left(1+\gamma_{l} \frac{\sum_{i=1}^{N}\left|g_{i l}\right|^{4}}{E_{l}^{2}}\right)
$$

Proof: Let $A_{2}=\frac{1}{N} \sum_{i=1}^{N} a_{i}^{2}$, and $A_{4}=\frac{1}{N} \sum_{i=1}^{N} a_{i}^{4}$. Then the SINR is given by:

$$
\gamma_{k}=\frac{p_{k} b_{k}^{2} A_{2}^{2}}{\sigma^{2} A_{2}+\frac{1}{N} A_{4} \sum_{l=k+1}^{K} p_{l} b_{l}^{2}}
$$

Define $\beta_{k}=\frac{p_{k} b_{k}^{2} A_{2}^{2}}{\gamma_{k}}$. Hence

$$
\begin{gathered}
\beta_{k}=\sigma^{2} A_{2}+\frac{1}{N} A_{4} \sum_{l=k+1}^{K} p_{l} b_{l}^{2} \\
\beta_{k-1}=\beta_{k}\left(1+\frac{1}{N} \gamma_{k} \frac{A_{4}}{A_{2}^{2}}\right)
\end{gathered}
$$

So it can be easily seen that the exchange of the decoding order of 2 users would not affect the remaining ones. Let us now consider two possible orderings $\left[\gamma_{k-1}, \gamma_{k}\right]$ (user $k-1$ is here decoded before user $k$ ) and $\left[\gamma_{k}, \gamma_{k-1}\right]$ with respective power allocations $\left[p_{k-1}, p_{k}\right]$ and $\left[p_{k}^{*}, p_{k-1}^{*}\right]$. Then:

$$
\begin{gathered}
p_{k}=\frac{\beta_{k} \gamma_{k}}{b_{k}^{2} A_{2}^{2}} \\
p_{k-1}=\frac{\beta_{k} \gamma_{k-1}}{b_{k-1}^{2} A_{2}^{2}}\left(1+\frac{1}{N} \gamma_{k} \frac{A_{4}}{A_{2}^{2}}\right) \\
p_{k-1}^{*}=\frac{\beta_{k} \gamma_{k-1}}{b_{k-1}^{2} A_{2}^{2}} \\
p_{k}^{*}=\frac{\beta_{k} \gamma_{k}}{b_{k}^{2} A_{2}^{2}}\left(1+\frac{1}{N} \gamma_{k-1} \frac{A_{4}}{A_{2}^{2}}\right)
\end{gathered}
$$

So that

$p_{k}+p_{k-1}-\left(p_{k}^{*}+p_{k-1}^{*}\right)=\frac{C}{b_{k-1}^{2}}-\frac{C}{b_{k}^{2}} \quad C=\frac{1}{N} \frac{\gamma_{k} \gamma_{k-1} A_{4}}{A_{2}^{4}}$

and the ordering to minimize the requested power depends only on the channel energies (since for user $k$, the energy is given by $E_{k}=b_{k}^{2} \sum_{i=1}^{N} a_{i}^{2}$ ) and therefore the decoding order should be done in terms of decreasing energies $\left(b_{k-1}^{2}>b_{k}^{2}\right)$. The result follows therefore directly.

\subsection{MMSE-SIC receiver}

The i.i.d. case had been dealt in [8]. In this section, we extend the results to a flat fading scenario, i.e. $g_{i k}=g_{k}$, where the SINR can be rewritten as

$$
\gamma_{k}=p_{k} \frac{\left|g_{k}\right|^{2}}{\sigma^{2}+\frac{1}{N} \sum_{l=k+1}^{K} \frac{p_{l}\left|g_{l}\right|^{2}}{1+\gamma_{l}}} .
$$

Result: For the case of flat fading channels, the optimal decoding ordering depends on the user requested SINR, weighted by the individual path losses, and follows the ordering $\frac{1+\gamma_{1}}{\left|g_{1}\right|^{2}}<\frac{1+\gamma_{2}}{\left|g_{2}\right|^{2}}<\cdots<\frac{1+\gamma_{K}}{\left|g_{K}\right|^{2}}$. Moreover, the power allocated to each user has an explicit form given by:

$$
p_{k}=\frac{\gamma_{k}}{\left|g_{k}\right|^{2}} \sigma^{2} \prod_{i=k+1}^{K}\left[1+\frac{1}{N} \frac{\gamma_{i}}{1+\gamma_{i}}\right] .
$$

Proof: The proof follows the same steps as the one in 4.1.

Further generalizations, as the separable model $g_{i k}=a_{i} b_{k}$ are still under study. However, for a high SNR regime, the SINR expression can be approximated by

$$
\gamma_{k}=\frac{p_{k}}{N} \sum_{i=1}^{N} \frac{\left|g_{i k}\right|^{2}}{\frac{1}{N} \sum_{l=k+1}^{K} \frac{p_{l}\left|g_{i l}\right|^{2}}{1+\gamma_{l}}}=p_{k} \frac{\left|b_{k}\right|^{2}}{\frac{1}{N} \sum_{l=k+1}^{K} \frac{p_{l}\left|b_{l}\right|^{2}}{1+\gamma_{l}}},
$$

so it boils down to eq.(4) with $b_{k}$ playing the role of $g_{k}$. As a consequence, the same result as for the flat fading holds.

\section{DISTRIBUTED ALLOCATION FOR MMSE-SIC}

In many cases, the central entity can not feedback to the users the different powers in order to satisfy the requested rates. Moreover, the downlink overhead signalling may dramatically impact the useful rate as the number of users in the system increases. In these cases, a decentralized approach may be used where each user determines solely his power. Previous attempts for the analysis of decentralized schemes rely on game theoretic approaches [12]. In this section, we will show how asymptotic analysis can be used in this setting.

\subsection{Distributed scheme}

We consider a system in which users have a discrete set of $\mathrm{M}$ different available rates to choose from $R_{1}, \ldots, R_{M}$, as is the case in UMTS or other wireless local area network standards. The number of users in each class rate is denoted by $K_{1}, \ldots, K_{M}$. The users are supposed to know the average fraction of users with a certain rate i.e $K_{i}^{*}=\operatorname{pr}\left(R=R_{i}\right) K$ as well as the total number of users $K$ in the system. The values $\operatorname{pr}\left(R=R_{i}\right)$ are usually provided by previous measurements on the user's system behavior. In the case of a high number of users, the following holds:

$$
K_{i} \approx K_{i}^{*}=\operatorname{pr}\left(R=R_{i}\right) K
$$




\subsection{Groupwise detection}

In the case of i.i.d signatures, a user in rate class $K_{m}$ can estimate his SINR and his decoding order since in this case, equation (4) boils down to:

$$
\gamma_{k} \approx p_{k} \frac{1}{\sigma^{2}+\frac{1}{N} \sum_{l=m}^{M} K_{m}^{*} \frac{p_{l}}{1+\gamma_{l}}}
$$

The receiver in this case needs to implement a Groupwise SIC. Indeed, it is not possible for the user to determine precisely in which order he will be decoded among all the users with the same rate requirements, since this decision can be taken arbitrarily by the base station. Users in the same class can be decoded either in an MMSE filter or MMSE SIC fashion. In the latter case, users will have a better SINR then the targeted one which will reduce the probability of error. Moreover, as previously, the power allocation has an explicit form which depends only on the probabilities of the users to be in a certain class:

$$
p^{k}=\gamma_{k} \sigma^{2}+\frac{1}{N} \sum_{l=m}^{M} K_{m}^{*} \frac{p_{l}}{1+\gamma_{l}} .
$$

For the MMSE-SIC, the groups of users should be decoded in order of increasing requested rates, by a derivation following the lines of the one in section 4.1. Note that the same does not hold for the matched filter as the decoding order depends on the channel strength (which is the same in the i.i.d case) and not the target SINR's.

\section{SIMULATIONS}

In this section, some numerical results are presented to illustrate the theoretical claims. All simulation have been performed for an $\mathrm{SNR}\left(\mathrm{SNR}=\frac{1}{\sigma^{2}}\right)$ of $10 \mathrm{~dB}$. Figure 1 presents the requested and achieved rates for the SIC matched filter with optimal power allocation and decoding order. The users share a common power profile along the different dimensions and are supposed to be affected by random path losses. As one can see, the asymptotic results match for a reasonable system with $N=256$ and $K=100$.

In figure 2, the required power for a set of requested rates is plotted for the MMSE and matched filter SIC for different loads: $\alpha=0.2$ and $\alpha=0.6$. An important gain is achieved with the MMSE-SIC filter, especially as the load increases.

In figures 3 and 4 , the achieved rates for the distributed power allocation scheme with a MMSE-SIC receiver are shown for $N=64$ and $K=30$ as well as $N=256$ and $K=100$. In the system, four available rates are considered (which are randomly requested by the users with equal probability). For each user, the requested and obtained rates for a certain channel realization are plotted. It can be seen that the results obtained are quite good already for a system with $N=64$ and
$K=30$ when the users know only the probabilities of the requested rates.

\section{CONCLUSIONS}

In this contribution, the optimal decoding order and power allocation has been derived for SIC receivers, both MMSE and matched filter, considering different, realistic channel models. Interestingly, we have shown that the use of asymptotic tools from random matrix theory provide a neat framework for the analysis of SIC systems. It has also been shown, that under certain conditions, the power allocation can be determined in a decentralized manner (by each user individually) when considering a high number of users in the network.

\section{REFERENCES}

[1] H. Boche and S. Stánczak, "Convexity of some feasible QoS regions and asymptotic behavior of the minimum total power in CDMA systems," IEEE Transactions on Communications, vol. 52, no. 12, pp. 2190-2197, Dec. 2004.

[2] E.A. Jorswieck, H. Boche, and A. Sezgin, "Delaylimited capacity and maximum throughput of spatially correlated multiple antenna systems under average and peak-power constraints," in IEEE Information Theory Workshop (ITW'04), 24-29 2004, pp. 440-445.

[3] E.A. Jorswieck and H. Boche, "Transmission strategies for the MIMO MAC with MMSE receiver: Average MSE optimization and achievable individual MSE region," IEEE Transactions on Signal Processing, vol. 51, no. 11, pp. 2872-2881, Nov. 2003.

[4] R. Müller, "Combining multiuser detection and coding: Promises and problems," in 2000 Conference on Information Sciences and Systems, Princeton University, 15172000.

[5] G. Caire, S. Guemghar, A. Roumy, and S. Verdú, "Maximizing the spectral efficiency of coded CDMA under successive decoding," IEEE Transactions on Information Theory, vol. 50, no. 1, pp. 152-164, Jan. 2004.

[6] F. Meshkati, H. V. Poor, C. Schwartz, and D. Guo, "A unified power control algorithm for multiuser detectors in large systems: Convergence and performance," Sept. 2005, in Proceedings of the 43rd Allerton Conference on Communications, Control and Computing, UrbanaChampaign, Ill, USA.

[7] H. Li and H.V. Poor, "Power allocation, decoding order and spectral efficiency of successive interference cancellation based multirate ds-cdma systems," in Proc. of IEEE Globecom 04, Dallas, USA, 29Nov.-3Dec. 2004, pp. 2828-32. 
[8] A. Suárez, R de Lacerda, M. Debbah, and N. LinhTrung, "Power allocation under quality of service constraints for uplink multi-user systems," in IEEE 10th Biennial Vietnam Conference on Radio \& Electronics (REV'06), 6-7 2006.

[9] C-N. Chuah, J. M. Kahn, D.N. Tse, and R. Valenzuela, "Capacity scaling in mimo wireless systems under correlated fading," IEEE Transactions on Information Theory, vol. 48, no. 3, pp. 637-50, 2002.

[10] V. L. Girko, "Theory of Stochastic Canonical Equations, Volumes I and II ," Kluwer Academic Publishers, Dordrecht, The Netherlands, 2001.

[11] A. Tulino, L. Li, and S. Verdu, "Spectral Efficiency of Multicarrier CDMA," IEEE Transactions on Information Theory, pp. 479-505, February 2005.

[12] N. Bonneau, M. Debbah, E. Altman, and A. Hjorungnes, "Wardrop equilibrium for cdma systems," Third Workshop on Resource Allocation in Wireless NETworks, Limassol, pp. Limassol, Cyprus, April 2007.

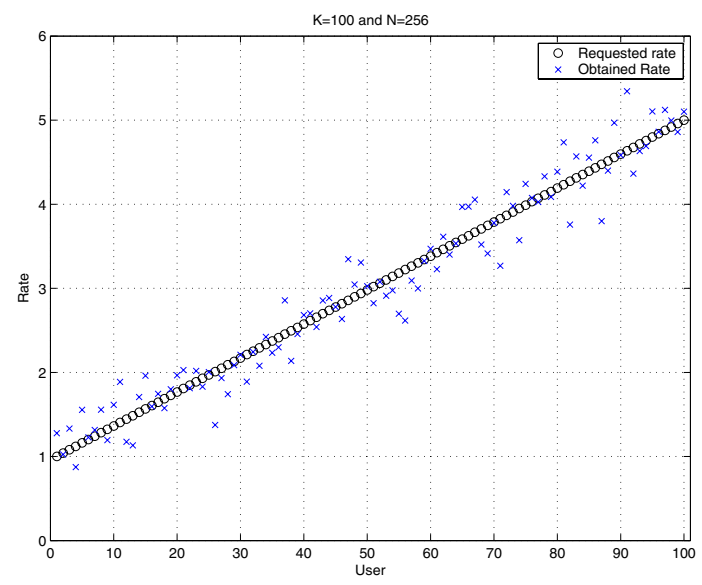

Fig. 1. Matched filter with $N=256$ and $K=100$ at 10dB.

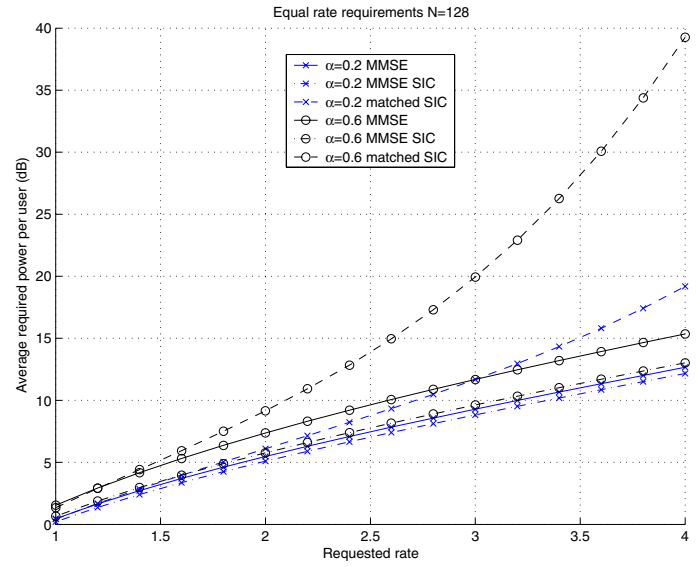

Fig. 2. Total power required for MMSE, matched filter SIC and MMSESIC with $N=128$ and respectively $\alpha=0.2, \quad \alpha=0.6$ at $10 \mathrm{~dB}$.

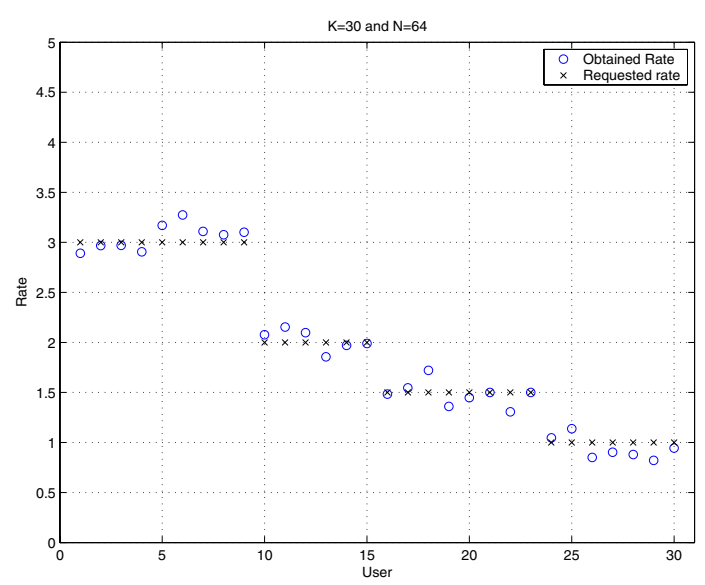

Fig. 3. Distributed power allocation for MMSE-SIC with $N=64$ and $K=30$ at $10 \mathrm{~dB}$.

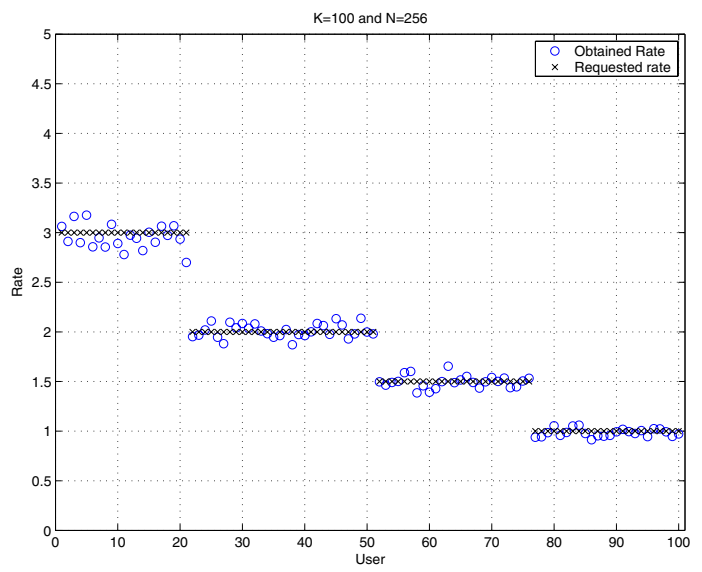

Fig. 4. Distributed power allocation for MMSE-SIC with $N=256$ and $K=100$ at $10 \mathrm{~dB}$. 